\title{
Estudo comparativo sobre consumo de madeira na construção de edificações convencionais e de alvenaria estrutural
}

A indústria da construção civil está entre as principais consumidoras de recursos naturais e geradoras de resíduos. Entre os principais recursos naturais utilizado no sistema convencional de execução em concreto armado está a madeira. Sendo que no final da execução da obra a madeira utilizada tem como destino final o aterro. Assim, este estudo objetivou realizar um comparativo com relação à madeira consumida em construção de edificações unifamiliares térreas convencionais e de alvenaria estrutural com o auxílio dos softwares TQS ${ }^{\circledR}$ e o Eberick ${ }^{\circledR}$ usando a sustentabilidade. A partir dos resultados dos cálculos referentes ao consumo de madeira nos dois sistemas construtivos, verificou-se que o sistema alvenaria estrutural é mais vantajoso para edificações com pano de lajes menores, mitigando o consumo de madeira em aproximadamente $80 \%$. Sendo assim, este sistema é uma excelente alternativa para o menor consumo de madeira, no entanto, seu melhor aproveitamento está condicionado a um projeto concebido com características necessárias, como a modulação, que é fácil implementação e a sensibilização dos clientes sobre um sistema construtivo que pode, além de trazer economia financeira, agir positivamente sobre o meio ambiente. Em ações sustentáveis em sistemas construtivos, praticadas por profissionais de engenharia e arquitetura, sensibilizados com os objetivos do desenvolvimento sustentáve nas construções enxutas para o próximo milênio proporcionarão benefícios econômicos, sociais e ambientais, fundamentais para uma gestão eficaz dos resíduos provenientes dos recursos naturais.

Palavras-chave: Sistemas Construtivos; Construção Enxuta; Sustentabilidade.

\section{Comparative study on wood consumption in the construction of conventional and structural masonry buildings}

\begin{abstract}
The construction industry is among the leading consumers of natural resources and waste generators. Among the main natural resources used in the conventional system of concrete execution, the reinforced one is in the wood. Since there is no final execution of the woodwork, use as final destination or landfill. Thus, this study aimed to make a comparison with respect to the wood consumed in the construction of uniform thermal, applicable buildings and structural masonry with the aid of TQS ${ }^{\circledast}$ and Eberick ${ }^{\circledR}$ software using sustainability. From the results of the calculations related to the wood consumption in two building systems, it was found that the structural masonry system is more advantageous for smaller background editions, reducing wood consumption by approximately $80 \%$. Thus, this system is an excellent alternative for lower wood consumption, however, its best use is conditional on a project designed with necessary features such as modulation, which is easy to use and customer awareness about a building system that can in addition to bringing financial savings, acting positively on the environment. Sustainable actions in construction systems, practiced by engineering and architecture professionals, sensitized with the objectives of sustainable development in grafted constructions for the next millennium, provided economic, social and environmental benefits, fundamental for an efficient management of natural resources residues.
\end{abstract}

Keywords: Constructive System; Lean Construction; Sustainability.

Topic: Construções e Arquitetura Sustentáveis

Reviewed anonymously in the process of blind peer
Received: 02/10/2020

Approved: $24 / 11 / 2020$
Yutaka Mario Kobayashi Júnior (iD)

Unicesumar, Brasil

http://lattes.cnpq.br/0913796918242414

http://orcid.org/0000-0003-2990-5340

yutaka_mkj@yahoo.com.br

\section{Cleiltan Novaes da Silva (id}

Unicesumar, Brasil

http://orcid.org/0000-0002-6194-1950

cleiltan.silva@unicesumar.edu.br

Luciana Cristina Soto Herek Rezende (iD)

Unicesumar, Brasil

http://lattes.cnpq.br/1158006438170059

http://orcid.org/0000-0001-9677-4139

luciana.resende@unicesumar.edu.br

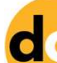

DOI: 10.6008/CBPC2179-6858.2020.006.0024

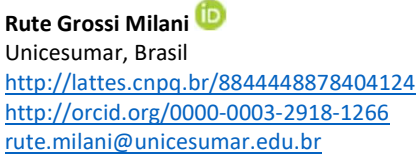

Edneia Aparecida de Souza Paccola (ib

Unicesumar, Brasi

http://lattes.cnpq.br/5090759600495959

http://orcid.org/0000-0002-3182-3224

edneia.paccola@gmail.com

Referencing this:

KOBAYASHI JÚNIOR, Y. M.; SILVA, C. N.; REZENDE, L. C. S. H.; MILANI, R. G.; PACCOLA, E. A. S.. Estudo comparativo sobre consumo de madeira na construção de edificações convencionais e de alvenaria estrutural. Revista Ibero Americana de Ciências Ambientais, v.11, n.6, p.284-297, 2020. DOI: http://doi.org/10.6008/CBPC2179-

6858.2020 .006 .0024 


\section{INTRODUÇÃO}

A cadeia da construção civil é reconhecida como uma das mais importantes atividades para o desenvolvimento econômico e social de um país. O setor é responsável por inúmeros empregos diretos e indiretos, além de exercer fundamental importância para a infraestrutura necessária ao crescimento e desenvolvimento de uma nação (KARPINSKI et al., 2009).

No entanto, apesar dos impactos socioeconômicos positivos, o setor da construção atualmente, enfrenta pressões relacionadas à diminuição de impactos ambientais e à melhoria de desempenho do ambiente construído (SILVA et al., 2014). As atividades envolvidas em processos de construção são as principais causas do esgotamento recursos naturais, representando $24 \%$ da extração de recursos naturais em escala global (BRIBÍAN et al., 2010) e eles também são os principais geradores de resíduos.

As construções demandam materiais, tais como areia e cascalho, que são comumente extraídos de sedimentos aluviais, modificando o perfil dos rios e o seu equilíbrio, acarretando problemas ambientais (BRASILEIRO et al., 2015). Além do esgotamento dos recursos naturais, a extração de matéria-prima que alimenta a indústria da construção civil provoca outros impactos ambientais que devem ser cuidadosamente considerados, como danos à paisagem, degradação do ecossistema, danos à saúde humana e a contaminação do solo, água e ar pelas emissões provenientes da produção e manuseio de materiais de construção (BLANKENDAAL et al., 2014).

Ademais, estudos apontam que o setor da construção civil é o principal agente que causa de emissões de Gases de Efeito Estufa (GEE) (KHASREEN et al., 2009). Muito embora, vários estudos apontem os impactos da contrução ao meio ambiente, a complexidade desse setor, devido as inúmeras cadeias envolvidas, tornase uma barreira para o alcance da sustenbilidade da construção civil.

No setor da construção civil, as cadeias de materiais de produção incluem a cadeia da madeira, das argilas e silicatos, do calcário, dos materiais químicos e petroquímicos, da siderurgia, da metalurgia de nãoferrosos, dos materiais elétricos e das máquinas e equipamentos (ABRAMAT, 2015), sendo que cada uma dessas cadeias é formada por vários setores, responsáveis pela produção de vários produtos. Dentre estas cadeias, destaca-se a cadeia da madeira, que envolve a extração vegetal, o comércio de produtos in natura e as serrarias, onde a madeira é serrada e trabalhada (ABRAMAT, 2015).

Na construção civil, a madeira é utilizada para diferentes finalidades, podendo ser em usos temporários (fôrmas para concreto, andaimes e escoramentos) ou de forma definitiva nas estruturas de cobertura, nas esquadrias, nos forros e nos pisos (CAPANEMA et al., 2013). Sendo que, as fontes de matéria prima de madeira podem ser de florestas plantadas ou nativas (CAPANEMA et al., 2013). Assim, a busca por tecnologias alternativas que diminuam o consumo de madeira e que viabilizem o avanço da construção aliado à conservação dos recursos naturais faz se necessária.

Além do grande volume e variedades de matérias primas de fontes renováveis e não renováveis necessários para atender a demanda do setor da construção, os resíduos oriundos do setor da construção e demolição, têm sido amplamente discutidos, devido aos impactos socioambientais ocasionados quando 
manejados inadequadamente.

Os resíduos gerados durante construção ou demolição $(R C D)$ constituem uma parte representativa do total de resíduos produzidos nas cidades, representando cerca de $40 \%$ a $60 \%$ dos resíduos sólidos urbanos (RSU) produzidos diariamente (SILVA et al., 2012), superando os resíduos domésticos.

Quando descartados inadequadamente, estes resíduos podem acarretar impactos ambientais, sociais e econômicos. O setor da construção civil responde por um volume significativo de resíduos depositados em locais de deposição irregulares nas cidades, como encostas de rios, vias, logradouros públicos e terrenos baldios (ALVES, 2015; PASCHOALIN FILHO et al., 2015). Desta forma, comprometem a paisagem urbana e dificultam o tráfego de pedestres e veículos (KARPINSKI et al., 2009), esgotam as áreas de aterros, prejudicam a drenagem urbana e contribuem com os alagamentos e enchentes (ULSEN et al., 2013), e compromete a saúde pública, pois servem de abrigo para a multiplicação de vetores transmissores de doenças (KARPINSKI et al., 2009).

Diante do contexto, o presente trabalho teve como objetivo realizar um comparativo em relação à madeira consumida em construção de edificações unifamiliares térreas convencionais e de alvenaria estrutural, por meio dos softwares para cálculo estrutural $T Q S^{\circ}$ e o Eberick ${ }^{\circledR}$ visando a sustentabilidade.

\section{MATERIAIS E MÉTODOS}

Local e época

Este estudo foi conduzido durante os anos de 2017 e 2018, e os dados foram obtidos a partir dos arquivos da empresa YK Engenharia, localizada no município de Maringá/PR. Os dados para realização dos cálculos foram coletados a partir do memorial descritivo de 30 edificações unifamiliares térreas construídas em sistema construtivo convencional, com áreas construídas variando entre 102,22 a 1.490,35 m².

\section{Cálculo da área média dos panos de lajes}

Para o cálculo da área média dos panos de lajes nas 30 edificações construídas em sistema convencional, foi realizada a soma da área de cada laje e dividido pela quantidade de lajes existentes no projeto.

\section{Determinação do comprimento total das vigas}

Para realização de um quantitativo preciso de escoras, foi medido o comprimento de todas as vigas do pavimento cobertura das 30 edificações analisadas.

\section{Cálculo do consumo de madeira nas edificações construídas em sistema convencional}

Para os cálculos de determinação da quantidade de madeira consumida nas trinta edificações construídas em sistema convencional, foram considerados: a área de forma das vigas e área de forma dos pilares foi quantificada as escoras de eucalipto (Eucalyptus grandis). 
Os resultados de área obtidos foram transformados em volume para determinar o volume total e a relação volume $X$ área, conforme as equações descritas abaixo.

Para o cálculo da área de forma das vigas, foi utilizada a equação 1.

$$
(A=(b w+2 \cdot h) \cdot L)
$$

Eq. 1

Em que: A é a área total de forma da peça; Bw é a largura da viga; h é a altura da viga; L é o comprimento da viga.

Para o cálculo da área de forma dos pilares, empregou-se a equação 2.

$$
(A=2 \cdot(a+b) \cdot L)
$$

Eq. 2

Em que:

A é a área total de forma da peça; A é a menor dimensão do pilar; B é a maior dimensão do pilar; L é a altura do pilar.

Para o cálculo de transformação da área de forma em volume de madeira, foi considerado uma espessura média de trinta e cinco milímetros $(35 \mathrm{~mm})$ das madeiras usadas para as formas, conforme a equação 3.

$$
(\text { Vol }=A \cdot e)
$$

Eq. 3

Em que:

Vol. é o volume total;

A é a área de forma total; E é a espessura da madeira ( $35 \mathrm{~mm})$.

\section{Quantificação das escoras de eucalipto (Eucalyptus grandis)}

Para quantificar as escoras foram consideradas as escoras das lajes e das vigas, com uma altura de três metros. Sendo utilizado uma por metro quadrado para lajes e para vigas, uma a cada metro para apoiálas.

\section{Quantificação das escoras de eucalipto para as lajes}

Para quantificar as escoras que apoiam as lajes para a concretagem, pegou-se o valor inteiro da área da edificação, uma vez que para o estudo está sendo considerado a utilização de uma escora para cada metro quadrado de laje.

\section{Quantificação das escoras de eucalipto para as vigas}

Para quantificar as escoras que apoiam as vigas para a concretagem, pegou-se o valor inteiro do comprimento total das vigas de cobertura, uma vez que para o estudo está sendo considerado a utilização de uma escora para cada metro de viga. 
Para o cálculo da transformação da quantidade de escoras de eucalipto e volume de madeira empregou-se a equação 4.

$$
\begin{array}{ll}
\left(\mathrm{Vol}=N \cdot \frac{\pi \cdot 0,10^{2}}{4} \cdot 3\right) & \text { Eq. } 4
\end{array}
$$

\section{Volume total e obtenção da relação volume por área}

Para o cálculo do volume total de madeira consumida nas edificações estudadas somou-se a Equação 3 com a Equação 4. Na sequência, para obtenção do índice médio de volume de formas de madeira por metro quadrado de obra executada, foi empregado a equação 5.

$$
\left(i=\frac{V o l}{A_{C}}\right)
$$

Em que:

Vol. é o volume total; Ac é a área de obra executada; i é a reação volume $x$ área.

\section{Simulação do consumo de madeira em sistemas construtivos de alvenaria convencional}

Para a simulação do consumo de madeira nas trinta edificações unifamiliares térreas construídas no sistema de alvenaria convencional, empregou-se os softwares para cálculo estrutural TQS ${ }^{\oplus}$ e o Eberick ${ }^{\circledR}$ Os softwares automatizaram o cálculo da área de forma utilizada para poder executar cada elemento estrutural, viga e pilar. O cálculo da área das formas foi realizado por área de peças retangulares, sendo as vigas com fechamento em três faces: duas laterais e o fundo, os pilares com fechamento nas quatro faces laterais.

\section{Agrupamento de faixas de obras e análise dos dados}

Para analisar o índice do consumo de madeira, as obras foram agrupadas em faixas de área média das lajes, sendo elas: ausência de lajes, de cinco a $10 \mathrm{~m}^{2}, 10,01$ a 15,00 m², 15,01 a 20,00 m² e acima de 20 $\mathrm{m}^{2}$. Os dados foram tabulados no programa Excel e analisados por meio de estatística descritiva. Além disso, foi utilizada uma análise de variância unifatorial (one way ANOVA) para testar a diferença no volume de madeira consumida $\left(\mathrm{m}^{3}\right)$ entre os sistemas de alvenaria convencional e estrutural. Para as análises, foram verificados os pressupostos de normalidade e homogeneidade de variâncias (Levene's test) e os valores foram considerados estatisticamente significantes em $p<0,05$. As análises foram realizadas no STATISTICA versão 7.1.

\section{RESULTADOS E DISCUSSÃO}

A avaliação média dos panos de laje e os comprimentos das vigas estão apresentados na tabela 1. Nota-se que as áreas médias das lajes não têm qualquer relação com a área das 30 edificações analisadas, 
observando que em edificações de área construída aproximada a concepção estrutural faz diferença para a quantidade e tamanhos das vigas.

Com as informações apresentadas (Tabela 1), compreende-se por meio dos cálculos da área média dos panos de lajes das edificações o modelo de concepção estrutural. Onde a obra se caracteriza por panos de lajes maiores, consequentemente com menor uso de forma e maior de escoramento de madeira para as lajes ou por panos de lajes menores, que representa uma quantidade maior de vigas e formas para caixarias e escoramento na obra.

Tabela 1: Apresentação das obras analisadas com valor da área da edificação $\left(\mathrm{m}^{2}\right)$, área de forma de madeira $\left(\mathrm{m}^{2}\right)$, área média das lajes $\left(\mathrm{m}^{2}\right)$ e comprimento das vigas da cobertura $(\mathrm{m})$.

\begin{tabular}{|c|c|c|c|c|}
\hline Edificação & $\begin{array}{l}\text { Área da edificação } \\
\left(m^{2}\right)\end{array}$ & $\begin{array}{l}\text { Área de Forma } \\
\left(\mathrm{m}^{2}\right)\end{array}$ & $\begin{array}{l}\text { Área média dos panos de lajes } \\
\left(\mathrm{m}^{2}\right)\end{array}$ & $\begin{array}{l}\text { Comprimento das vigas de cobertura } \\
\text { (m) }\end{array}$ \\
\hline 1 & 102,22 & 292,83 & 12,55 & 80 \\
\hline 2 & 129,07 & 315,33 & 10,32 & 108 \\
\hline 3 & 135,4 & 354,5 & 12,95 & 103 \\
\hline 4 & 141,9 & 326,6 & 9,36 & 133 \\
\hline 5 & 146,24 & 322,78 & 13,14 & 108 \\
\hline 6 & 146,46 & 441,29 & 0 & 143 \\
\hline 7 & 147,62 & 274,48 & 0 & 134 \\
\hline 8 & 150 & 233,06 & 19,76 & 88 \\
\hline 9 & 164,09 & 362,6 & 8,68 & 161 \\
\hline 10 & 165,85 & 645,53 & 8,07 & 152 \\
\hline 11 & 180,96 & 413,37 & 13 & 173 \\
\hline 12 & 192,71 & 487,46 & 19,13 & 197 \\
\hline 13 & 199 & 636,78 & 6,63 & 191 \\
\hline 14 & 199,12 & 644,31 & 12,65 & 259 \\
\hline 15 & 217,17 & 284,5 & 33,16 & 79 \\
\hline 16 & 225,46 & 881,84 & 12,2 & 259 \\
\hline 17 & 226,7 & 652,89 & 8,68 & 161 \\
\hline 18 & 236,81 & 491,26 & 11,73 & 217 \\
\hline 19 & 238,92 & 625,56 & 26,6 & 132 \\
\hline 20 & 243,44 & 692,01 & 9 & 150 \\
\hline 21 & 261,41 & 636,15 & 16,7 & 187 \\
\hline 22 & 270,81 & 569,26 & 14,27 & 210 \\
\hline 23 & 287,41 & 489,41 & 26 & 166 \\
\hline 24 & 292,41 & 723,65 & 16 & 489 \\
\hline 25 & 352 & 478,12 & 35 & 145 \\
\hline 26 & 380,12 & 1155,66 & 10,34 & 336 \\
\hline 27 & 468,98 & 677,23 & 19,7 & 217 \\
\hline 28 & 695,14 & 488,99 & 0 & 127 \\
\hline 29 & 1470,48 & 4195,15 & 24,3 & 974 \\
\hline 30 & 1490,35 & 3228,52 & 30 & 851 \\
\hline
\end{tabular}

Com o cálculo da área média das lajes e do comprimento total das vigas nas trinta edificações (Tabela 1) foi determinado o consumo de madeira para cada edificação com o objetivo de mensurar o volume desta consumida nas formas, no escoramento de lajes e vigas. Com a separação destes elementos, de acordo com a função destes na obra, foi possível observar o volume que cada sistema estrutural consome de madeira na etapa construtiva em cada edificação (Tabela 2).

Tabela 2: Cálculo do volume (vol.) de madeira gerada por cada edificação analisada.

\begin{tabular}{llll}
\hline Edificação & $\begin{array}{l}\text { Vol. de madeira } \\
\text { formas }\left(\mathbf{m}^{\mathbf{3}}\right)\end{array}$ & $\begin{array}{l}\text { para } \\
\text { laje }\left(\mathbf{m}^{\mathbf{3}}\right)\end{array}$ & $\begin{array}{l}\text { Vol. de madeira para o escoramento } \\
\text { viga }\left(\mathbf{m}^{\mathbf{3}}\right)\end{array}$ \\
\hline 1 & 10,25 & 2,40 & 1,88 \\
2 & 11,04 & 3,04 & 2,54 \\
3 & 12,41 & 3,18 & 2,43 \\
4 & 11,43 & 3,32 & 3,13
\end{tabular}




\begin{tabular}{|c|c|c|c|}
\hline 5 & 11,30 & 3,44 & 2,54 \\
\hline 6 & 15,45 & 0,00 & 3,37 \\
\hline 7 & 9,61 & 0,00 & 3,16 \\
\hline 8 & 8,16 & 3,53 & 2,07 \\
\hline 9 & 12,69 & 3,86 & 3,79 \\
\hline 10 & 22,59 & 3,89 & 3,58 \\
\hline 11 & 14,47 & 4,24 & 4,08 \\
\hline 12 & 17,06 & 4,52 & 4,64 \\
\hline 13 & 22,29 & 4,69 & 4,50 \\
\hline 14 & 22,55 & 4,69 & 6,10 \\
\hline 15 & 9,96 & 5,11 & 1,86 \\
\hline 16 & 30,86 & 5,30 & 6,10 \\
\hline 17 & 22,85 & 5,32 & 3,79 \\
\hline 18 & 17,19 & 5,56 & 5,11 \\
\hline 19 & 21,89 & 5,61 & 3,11 \\
\hline 20 & 24,22 & 5,73 & 3,53 \\
\hline 21 & 22,27 & 6,15 & 4,41 \\
\hline 22 & 19,92 & 6,36 & 4,95 \\
\hline 23 & 17,13 & 6,76 & 3,91 \\
\hline 24 & 25,33 & 6,88 & 11,52 \\
\hline 25 & 16,73 & 8,29 & 3,42 \\
\hline 26 & 40,45 & 8,95 & 7,92 \\
\hline 27 & 23,70 & 11,03 & 5,11 \\
\hline 28 & 17,11 & 0,00 & 2,99 \\
\hline 29 & 146,83 & 34,64 & 22,95 \\
\hline 30 & 113,00 & 35,11 & 20,05 \\
\hline Índice Médio & 25,69 & 6,72 & 5,29 \\
\hline Porcentagem (\%) & 68,15 & 17,83 & 14,02 \\
\hline
\end{tabular}

Paiva Filho et al. (2018) apresentou que $100 \%$ das empresas utilizam madeira para formas de elementos de concreto e que $25 \%$ utilizam a madeira como forma de escoramento. Como apresentado na Tabela 2, mesmo utilizando a alvenaria estrutural verifica-se a permanecia de um volume de madeira para o escoramento das lajes.

Os resultados dos cálculos da área de forma (vigas e pilares), quantidade de escoras de eucalipto, volume total de madeira consumida e o índice de relação volume por área $\left(\mathrm{m}^{2} / \mathrm{m}^{2}, \mathrm{~m}^{3} / \mathrm{m}^{2}\right)$ nas 30 edificações unifamiliares térreas construídas no sistema convencional, estão descritos na Tabela 3. Percebe-se que os volumes de madeira consumida nas edificações variam, não apresentando uma relação direta com as áreas das construções.

No âmbito dos sistemas construtivos, Santiago (2008) menciona que em edificações no sistema convencional ocorre um grande desperdício de material utilizado, a falta de padronização da execução do trabalho, dificuldade na fiscalização, controle de qualidade dos serviços prestados e a carência de um planejamento adequado no momento da execução.

Por intermédio da análise dos dados de obras de alvenaria estrutural e de concreto armado, Silva et al. (2017) salientam que a alvenaria estrutural apresentou menores gastos com fôrmas, mão de obra industrializada e cimbramento metálico, além de seu custo final para a execução retratar maiores economias em relação ao de concreto armado.

Ressaltar-se que um dos problemas relacionados com o uso da madeira, é a extração de toras para a obtenção de produtos, pois o ciclo se inicia com o de desmatamento (INPE, 2008), em que a construção civil um dos principais mercados consumidores da madeira (ZENID, 2009). Além disso, apesar do número ser incerto, estima-se que $80 \%$ da madeira nativa amazônica comercializada tenha origem ilegal (ZENID, 2009). 
Diante dessa problemática a busca por tecnologias alternativas para otimizar os sistemas construtivos perante ao uso da madeira se intensificou nos últimos anos. Além da economia do consumo de madeira, a alvenaria estrutural apresenta vantagens relacionadas com o custo (KATO, 2014; ROMAN et al., 1999).

Tabela 3: Área das edificações e resultado da área de forma (vigas e pilares), quantidade de escoras de eucalipto, volume total de madeira consumida e o índice de relação por área nas trinta edificações unifamiliares térreas construídas no sistema convencional.

\begin{tabular}{|c|c|c|c|c|c|c|}
\hline \multirow[t]{2}{*}{ Edificações } & \multirow{2}{*}{$\begin{array}{l}\text { Área das } \\
\text { edificações }\left(\mathrm{m}^{2}\right)\end{array}$} & \multirow{2}{*}{$\begin{array}{l}\text { Área de } \\
\text { forma }\left(m^{2}\right)\end{array}$} & \multirow{2}{*}{$\begin{array}{ll}\text { Quantidade } & \text { de } \\
\text { escoras (unidade) } & \end{array}$} & \multirow{2}{*}{$\begin{array}{l}\text { Volume de madeira } \\
\text { consumida }\left(\mathrm{m}^{3}\right)\end{array}$} & \multicolumn{2}{|c|}{$\begin{array}{l}\text { Índice de relação } \\
\text { madeira por área }\end{array}$} \\
\hline & & & & & $\left(m^{2} / m^{2}\right)$ & $\left(\mathrm{m}^{3} / \mathrm{m}^{2}\right)$ \\
\hline 1 & 102,22 & 292,83 & 182 & 14,54 & 2,86 & 0,14 \\
\hline 2 & 129,07 & 315,33 & 237 & 16,62 & 2,44 & 0,13 \\
\hline 3 & 135,40 & 354,5 & 238 & 18,02 & 2,62 & 0,13 \\
\hline 4 & 141,90 & 326,6 & 274 & 17,89 & 2,30 & 0,13 \\
\hline 5 & 146,24 & 322,78 & 254 & 17,28 & 2,21 & 0,12 \\
\hline 6 & 146,46 & 441,29 & 289 & 18,81 & 3,01 & 0,13 \\
\hline 7 & 147,62 & 274,48 & 281 & 12,76 & 1,86 & 0,09 \\
\hline 8 & 150,00 & 233,06 & 238 & 13,76 & 1,55 & 0,09 \\
\hline 9 & 164,09 & 362,6 & 325 & 20,35 & 2,21 & 0,12 \\
\hline 10 & 165,85 & 645,53 & 317 & 30,06 & 3,89 & 0,18 \\
\hline 11 & 180,96 & 413,37 & 353 & 22,79 & 2,28 & 0,13 \\
\hline 12 & 192,71 & 487,46 & 389 & 26,23 & 2,53 & 0,14 \\
\hline 13 & 199,00 & 636,78 & 390 & 31,48 & 3,20 & 0,16 \\
\hline 14 & 199,12 & 644,31 & 458 & 33,34 & 3,24 & 0,17 \\
\hline 15 & 217,17 & 284,5 & 296 & 16,93 & 1,31 & 0,08 \\
\hline 16 & 225,46 & 881,84 & 484 & 42,27 & 3,91 & 0,19 \\
\hline 17 & 226,70 & 652,89 & 387 & 31,97 & 2,88 & 0,14 \\
\hline 18 & 236,81 & 491,26 & 453 & 27,87 & 2,07 & 0,12 \\
\hline 19 & 238,92 & 625,56 & 370 & 30,61 & 2,62 & 0,13 \\
\hline 20 & 243,44 & 692,01 & 393 & 33,48 & 2,84 & 0,14 \\
\hline 21 & 261,41 & 636,15 & 448 & 32,82 & 2,43 & 0,13 \\
\hline 22 & 270,81 & 569,26 & 480 & 31,23 & 2,10 & 0,12 \\
\hline 23 & 287,41 & 489,41 & 453 & 27,80 & 1,70 & 0,10 \\
\hline 24 & 292,41 & 723,65 & 781 & 43,73 & 2,47 & 0,15 \\
\hline 25 & 352,00 & 478,12 & 497 & 28,44 & 1,36 & 0,08 \\
\hline 26 & 380,12 & 1155,66 & 716 & 57,32 & 3,04 & 0,15 \\
\hline 27 & 468,98 & 677,23 & 685 & 39,84 & 1,44 & 0,08 \\
\hline 28 & 695,14 & 488,99 & 822 & 20,11 & 0,70 & 0,03 \\
\hline 29 & 1470,48 & 4195,15 & 2444 & 204,42 & 2,85 & 0,14 \\
\hline 30 & 1490,35 & 3228,52 & 2341 & 168,16 & 2,17 & 0,11 \\
\hline $\begin{array}{l}\text { Índice } \\
\text { Médio }\end{array}$ & 318,61 & 734,04 & 542,5 & 37,70 & 2,40 & 0,12 \\
\hline
\end{tabular}

Notou-se que os índices de relação volume madeira não apresentaram uma correlação confiável com as áreas das trinta edificações, que variaram $0,7 \mathrm{~m}^{2} / \mathrm{m}^{2}$ a $3,91 \mathrm{~m}^{2} / \mathrm{m}^{2}$, devido à concepção estrutural, por isso foi necessária a realização dos cálculos das áreas médias dos panos de lajes.

De acordo com Mohamad et al. (2015) a alvenaria estrutural está sendo largamente utilizada como sistema construtivo capaz de suprir a demanda por habitação, atendendo aos critérios de desempenho e custo. Segundo os mesmos autores, esse sistema construtivo tem muitas outras vantagens, mas a econômica é uma das principais, devido à otimização de tarefas na obra, por meio de técnicas executivas simplificadas e facilidade de controle nas etapas de produção e eliminação de interferências, o que gera diminuição no desperdício de materiais produzidos pelo constante retrabalho.

Trabalho realizado por Geraldo et al. (2017), comparando dois sistemas para a construção de um edifício popular, observaram a economia de aproximadamente $20 \%$ quando utilizado o sistema estrutural 
em relação ao convencional. Os autores ressaltaram como vantagens mais imediatas da alvenaria estrutural a redução de custos de mão de obra e material, além da economia de custo em relação à diminuição do prazo de execução.

Dessa forma, o sistema construtivo de alvenaria estrutural torna-se um método viável para minimizar o consumo de madeira pela construção civil. Atrelado a isso, a reciclagem e o reaproveitamento de RCD podem contribuir para a sustentabilidade do setor na medida em que minimizam a geração de resíduos e o consumo de matéria-prima não renovável, reduzindo os impactos ambientais advindos das atividades de extração de matéria-prima e disposição final dos resíduos (BOHNENBERGER et al., 2018).

Segundo Carmo et al. (2012), o beneficiamento e posterior utilização dos resíduos de construção civil tem sido visto como uma alternativa para o fechamento do seu ciclo produtivo, mostrando-se ambientalmente viável tanto através da incorporação desses rejeitos em novos produtos como também para substituição de material.

Neste sentido, Murta et al. (2010), avaliaram três soluções estruturais para uma habitação unifamiliar portuguesa, sendo denominadas de solução tradicional (pilar-viga de betão armado), solução sustentável I (solução estrutural à base de materiais naturais), solução sustentável II (contempla a reutilização de madeira proveniente de demolições habitacionais). Os autores verificaram, que em termos de custo e vantagens ambientais construção, II foi mais vantajosa.

Na figura 1, é possível observar que, de acordo com o lançamento estrutural, quanto maior a área média das lajes na construção, ou seja, menor a quantidade de vigas, a economia da madeira será menor quando utilizado o sistema em alvenaria estrutural, uma vez que as lajes são mantidas. Observa-se um desvio padrão maior com o aumento das áreas médias dos panos de laje.

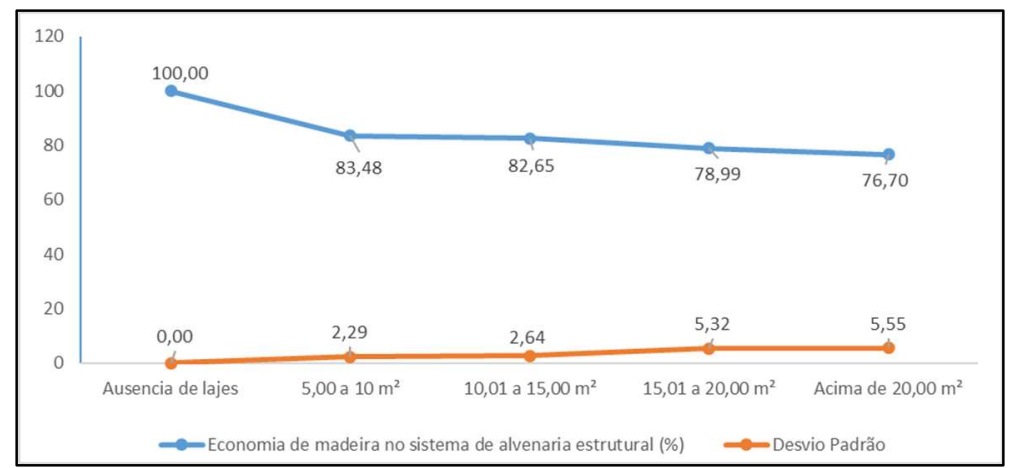

Figura 1: Comportamento dos índices de economia na geração de madeira e do desvio padrão, considerando o agrupamento das edificações.

Também é possível verificar que as construções em alvenaria estrutural são mais vantajosas e sustentáveis para obras consideradas convencionais, que são sistemas construtivos sem lajes ou com pequenos panos de lajes. Os dados somam ao que Cruz et al. (2018) demonstrou: a alvenaria estrutural como um processo mais econômico diminuindo as etapas construtivas de uma obra em comparação com o sistema convencional.

Analisando a figura 1, sobre o comportamento da economia de madeira nas edificações, foi proposto o agrupamento das edificações através das áreas médias dos panos de laje, com a finalidade de obter um 
índice médio de consumo de madeira por $\mathrm{m}^{2}$ de construção.

Identifica-se que quanto maior a área média dos panos de lajes, maior será o desvio padrão, observando uma menor economia de madeira no sistema em alvenaria estrutural.

O resultado dos cálculos de volume total de madeira consumida nas 30 edificações construídas no sistema convencional (Tabela 4) variou entre 12,76 a 204,42 $\mathrm{m}^{3}$, com média de $37,70 \mathrm{~m}^{3}$. Valor considerado alto quando comparado com os dados obtidos mediante à simulação da construção de edificações no sistema construtivo de alvenaria estrutural (Tabela 4), cuja variação foi entre 0,00 a 35,11 $\mathrm{m}^{3}$, com média de 6,72 $\mathrm{m}^{3}$.

Tabela 4: Cálculo do volume de madeira, considerando o sistema convencional e o sistema de alvenaria estrutural.

\begin{tabular}{|c|c|c|c|}
\hline \multirow[b]{2}{*}{ Edificações } & \multirow[b]{2}{*}{ Área das edificações $\left(\mathrm{m}^{2}\right)$} & \multicolumn{2}{|c|}{ Volume de madeira consumida $\left(\mathrm{m}^{3}\right)$} \\
\hline & & Sistema Convencional & $\begin{array}{l}\text { Sistema de Alvenaria } \\
\text { Estrutural }\end{array}$ \\
\hline 1 & 102,22 & 14,54 & 2,40 \\
\hline 2 & 129,07 & 16,62 & 3,04 \\
\hline 3 & 135,40 & 18,02 & 3,18 \\
\hline 4 & 141,90 & 17,89 & 3,32 \\
\hline 5 & 146,24 & 17,28 & 3,44 \\
\hline 6 & 146,46 & 18,81 & 0,00 \\
\hline 7 & 147,62 & 12,76 & 0,00 \\
\hline 8 & 150,00 & 13,76 & 3,53 \\
\hline 9 & 164,09 & 20,35 & 3,86 \\
\hline 10 & 165,85 & 30,06 & 3,89 \\
\hline 11 & 180,96 & 22,79 & 4,24 \\
\hline 12 & 192,71 & 26,23 & 4,52 \\
\hline 13 & 199,00 & 31,48 & 4,69 \\
\hline 14 & 199,12 & 33,34 & 4,69 \\
\hline 15 & 217,17 & 16,93 & 5,11 \\
\hline 16 & 225,46 & 42,27 & 5,30 \\
\hline 17 & 226,70 & 31,97 & 5,32 \\
\hline 18 & 236,81 & 27,87 & 5,56 \\
\hline 19 & 238,92 & 30,61 & 5,61 \\
\hline 20 & 243,44 & 33,48 & 5,73 \\
\hline 21 & 261,41 & 32,82 & 6,15 \\
\hline 22 & 270,81 & 31,23 & 6,36 \\
\hline 23 & 287,41 & 27,80 & 6,76 \\
\hline 24 & 292,41 & 43,73 & 6,88 \\
\hline 25 & 352,00 & 28,44 & 8,29 \\
\hline 26 & 380,12 & 57,32 & 8,95 \\
\hline 27 & 468,98 & 39,84 & 11,03 \\
\hline 28 & 695,14 & 20,11 & 0,00 \\
\hline 29 & 1470,48 & 204,42 & 34,64 \\
\hline 30 & 1490,35 & 168,16 & 35,11 \\
\hline Índice Médic & & 37,70 & 6,72 \\
\hline Porcentagen & & 0,85 & 0,15 \\
\hline
\end{tabular}

O índice da redução do consumo de madeira nas edificações no sistema construtivo de alvenaria estrutural variou de 69,80\% a 100\% conforme demonstrado na Tabela 5. Essa economia considera o consumo de madeira que ainda é utilizado mesmo no sistema de alvenaria estrutural, devido à presença de escoras para lajes; em obras cuja economia atingiu o valor de $100 \%$ representa, portanto, obras que não tem a presença de lajes.

A análise de variância evidenciou diferenças significativamente maiores no volume de madeira consumida $\left(\mathrm{m}^{3}\right)$ no sistema convencional em relação ao sistema de alvenaria estrutural (ANOVA: $F=15,80$; $p=0,0001$ ) (Figura 2). 


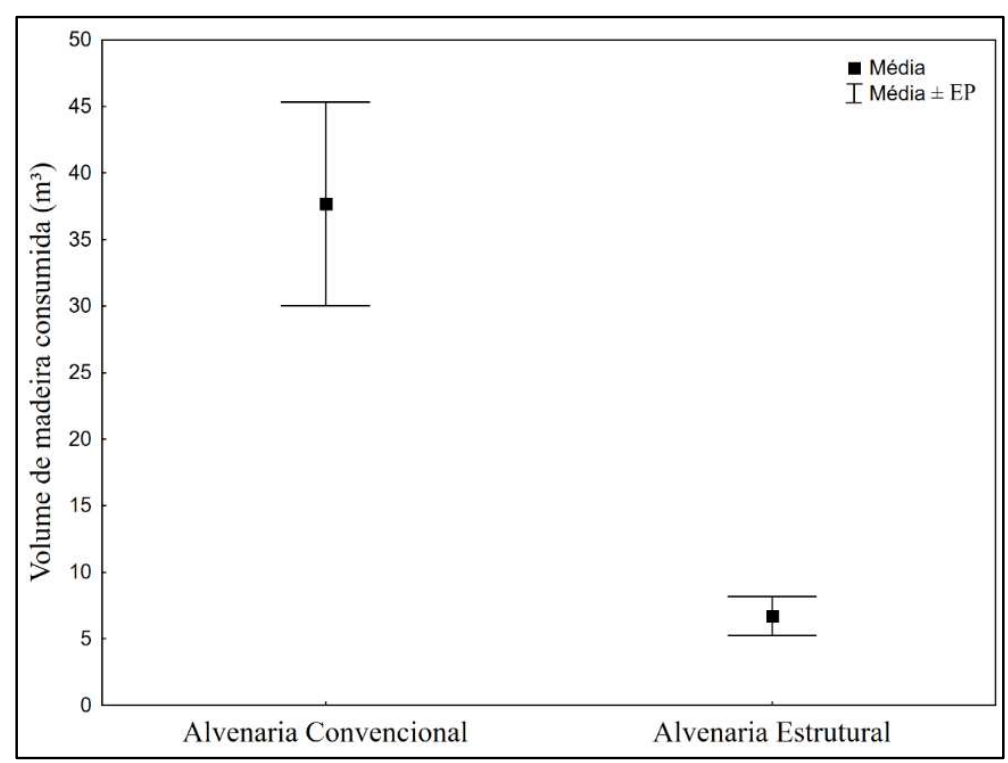

Figura 2: Valores médios do volume de madeira consumida $\left(\mathrm{m}^{3}\right)$ nos sistemas de alvenaria convencionais e estrutural. EP: erro padrão.

Tabela 5: Cálculo da economia do consumo de madeira no sistema de alvenaria estrutural.

\begin{tabular}{|c|c|}
\hline OBRA & Economia de madeira no sistema de alvenaria estrutural (\%) \\
\hline 1 & 83,47 \\
\hline 2 & 81,71 \\
\hline 3 & 82,34 \\
\hline 4 & 81,43 \\
\hline 5 & 80,09 \\
\hline 6 & 100,00 \\
\hline 7 & 100,00 \\
\hline 8 & 74,32 \\
\hline 9 & 81,01 \\
\hline 10 & 87,07 \\
\hline 11 & 81,39 \\
\hline 12 & 82,75 \\
\hline 13 & 85,10 \\
\hline 14 & 85,94 \\
\hline 15 & 69,80 \\
\hline 16 & 87,46 \\
\hline 17 & 83,34 \\
\hline 18 & 80,05 \\
\hline 19 & 81,68 \\
\hline 20 & 82,90 \\
\hline 21 & 81,26 \\
\hline 22 & 79,63 \\
\hline 23 & 75,68 \\
\hline 24 & 84,27 \\
\hline 25 & 70,84 \\
\hline 26 & 84,38 \\
\hline 27 & 72,32 \\
\hline 28 & 100,00 \\
\hline 29 & 83,06 \\
\hline 30 & 79,12 \\
\hline Índice Médio & 82,75 \\
\hline
\end{tabular}

Analisando o índice médio da redução do consumo de madeira nas trinta edificações (Tabela 5), notou-se que o sistema em alvenaria estrutural apresenta vantagens econômicas e ambientais, uma vez que houve uma redução média de $82,75 \%$ da madeira consumida pelo sistema construtivo convencional. Embasado nesse conceito, Mascarenhas et al. (2013), destaca que a adoção de práticas sustentáveis, além de promover o bem social, ambiental pode trazer o bem econômico através da redução de custos no 
processo produtivo. Avaliando os resultados da Tabela 5, a redução de madeira poderia ser maior desde que fossem desconsideradas as escoras de madeira, algo que, segundo Paiva Filho (2018) já acontece com a maioria das empresas, onde $75 \%$ das empresas não fazem uso de escoras de madeira, substituindo-as por escoras metálicas.

Observa-se na Tabela 6 que a geração de resíduo de madeira em uma obra varia no sistema convencional de 0,03 a $0,19 \mathrm{~m}^{3} / \mathrm{m}^{2}$ com média de $0,12 \mathrm{~m}^{3} / \mathrm{m}^{2}$ e no sistema de alvenaria estrutural de 0,000 a $0,024 \mathrm{~m}^{3} / 2$ com média de $0,021 \mathrm{~m}^{3} / \mathrm{m}^{2}$, sendo inviável fixar, portanto um índice absoluto. Propõe-se a possibilidade da utilização de índices por tipo de concepção estrutural, neste estudo, utilizando como parâmetro a área média das lajes.

Tabela 6: Cálculo do volume de madeira por metro quadrado de obra construída.

\begin{tabular}{|c|c|c|c|}
\hline \multirow[b]{2}{*}{ Edificações } & \multirow[b]{2}{*}{ Área das edificações $\left(\mathrm{m}^{2}\right)$} & \multicolumn{2}{|c|}{ Índice de relação volume por área $\left(\mathrm{m}^{3} / \mathrm{m}^{2}\right)$} \\
\hline & & Sistema Convencional & $\begin{array}{l}\text { Sistema de Alvenaria } \\
\text { Estrutural }\end{array}$ \\
\hline 1 & 102,22 & 0,14 & 0,024 \\
\hline 2 & 129,07 & 0,13 & 0,024 \\
\hline 3 & 135,40 & 0,13 & 0,023 \\
\hline 4 & 141,90 & 0,13 & 0,023 \\
\hline 5 & 146,24 & 0,12 & 0,024 \\
\hline 6 & 146,46 & 0,13 & 0,000 \\
\hline 7 & 147,62 & 0,09 & 0,000 \\
\hline 8 & 150,00 & 0,09 & 0,024 \\
\hline 9 & 164,09 & 0,12 & 0,024 \\
\hline 10 & 165,85 & 0,18 & 0,023 \\
\hline 11 & 180,96 & 0,13 & 0,023 \\
\hline 12 & 192,71 & 0,14 & 0,023 \\
\hline 13 & 199,00 & 0,16 & 0,024 \\
\hline 14 & 199,12 & 0,17 & 0,024 \\
\hline 15 & 217,17 & 0,08 & 0,024 \\
\hline 16 & 225,46 & 0,19 & 0,024 \\
\hline 17 & 226,70 & 0,14 & 0,023 \\
\hline 18 & 236,81 & 0,12 & 0,023 \\
\hline 19 & 238,92 & 0,13 & 0,023 \\
\hline 20 & 243,44 & 0,14 & 0,024 \\
\hline 21 & 261,41 & 0,13 & 0,024 \\
\hline 22 & 270,81 & 0,12 & 0,023 \\
\hline 23 & 287,41 & 0,10 & 0,024 \\
\hline 24 & 292,41 & 0,15 & 0,024 \\
\hline 25 & 352,00 & 0,08 & 0,024 \\
\hline 26 & 380,12 & 0,15 & 0,024 \\
\hline 27 & 468,98 & 0,08 & 0,024 \\
\hline 28 & 695,14 & 0,03 & 0,000 \\
\hline 29 & 1470,48 & 0,14 & 0,024 \\
\hline 30 & 1490,35 & 0,11 & 0,024 \\
\hline \multicolumn{2}{|c|}{ Índice Médio } & 0,12 & 0,021 \\
\hline \multicolumn{2}{|c|}{ Porcentagem (\%) } & 0,85 & 0,15 \\
\hline
\end{tabular}

Comparando esses valores com as porcentagens de outros estudos, Miranda (2009) descreve uma parcela de madeira nos resíduos de construção e demolição (RCD) na ordem de $42 \%$ e Tessaro et al. (2012) na ordem de $2 \%$. Como a prática da utilização de formas e escoras de madeira é muito utilizada pelas empresas, conforme cita Paiva Filho (2018), acredita-se que uma grande quantidade de madeira acaba sendo incinerada no local da obra, por muitas vezes, não ser possíveis o reaproveitamento, ter custos para o descarte e ser considerada uma prática comum, especialmente para as obras de pequeno porte. 


\section{CONCLUSÕES}

O sistema em alvenaria estrutural é uma excelente alternativa para o menor consumo de madeira, no entanto, um melhor aproveitamento desse sistema construtivo está condicionado a um projeto concebido com as características necessárias a esse sistema, como a modulação, de fácil implementação e a sensibilização dos clientes sobre um sistema construtivo que pode, além de trazer economia financeira, agir positivamente sobre o meio ambiente.

Salienta-se que ações sustentáveis em sistemas construtivos, praticadas por profissionais de engenharia e arquitetura, sensibilizados com os objetivos do desenvolvimento sustentável nas construções enxutas para o próximo milênio proporcionarão benefícios econômicos, sociais e ambientais, fundamentais para uma gestão eficaz dos resíduos provenientes dos recursos naturais.

\section{REFERÊNCIAS}

ABRAMAT. Associação Brasileira da Indústria de Materiais de Construção. Perfil da indústria de material de construção. Fundação Getúlio Vargas, 2015.

ALVES, J.. Resíduos da construção civil em obras novas. Interfaces Científicas-Exatas e Tecnológicas, v.1, n.1, p.5365, 2015.

BLANKENDAAL, T.; SCHUUR, P.; VOORDIJK H.. Reducing the environmental impact of concrete and asphalt: a scenario approach. Journal of Cleaner Production, v.66, p.27-36, 2014. DOI: https://doi.org/10.1016/j.jclepro.2013.10.012

BOHNENBERGER, J. C.; PIMENTA, J. F. P.; ABREU, M. V. S.; COMINI, U. B.; CALIJURI, M. L.; MORAES, A. P.; PEREIRA, I. S. . Identificação de áreas para implantação de usina de reciclagem de resíduos da construção e demolição com uso de análise multicritério. Ambiente Construído, v.18, n.1, p.299-311, 2018. DOI: https://doi.org/10.1590/s1678$\underline{86212018000100222}$

BRASILEIRO, L. L.; MATOS, J. M. E.. Revisão bibliográfica: reutilização de resíduos da construção e demolição na indústria da construção civil. Cerâmica, v.61, n.358, p.178189, 2015. DOI: https://doi.org/10.1590/0366$\underline{69132015613581860}$

BRIBÍAN, I. Z.; CAPILLA, A. V.; USÓN A. A.. Life cycle assessment of building materials: comparative analysis of energy and environmental impacts and evaluation of the eco efficiency improvement potential. Building and Environment, n.46, p.1133-1140, 2010. DOI: https://doi.org/10.1016/j.buildenv.2010.12.002

CAPANEMA, D. C. O.; GALVÃO, F. H.; SANTANA, K. M. F.; LUPP, L. S. L.; ALEXANDRE, R. A. S.; COSTA, T. A. R.. Setor Madeireiro e as Consequências do Uso da Madeira na Construção Civil: Possíveis Soluções. Percurso Acadêmico, v.3, n.6, 2013. DOI: https://doi.org/10.5752/P.22360603.2013v0n0p335-347

CARMO, D. S.; MAIA, N. S.; CÉSAR, C. G.. Avaliação da tipologia dos resíduos de Construção Civil entregues nas usinas de beneficiamento de Belo Horizonte. Revista Engenharia Sanitária Ambiental, v.17, n.2, p.187-192, 2012.
CRUZ, H. M.; SANTOS, D. G.; MENDES, L. A.. Causas da Variabilidade do tempo de execução dos processos em diferentes sistemas construtivos. Ambiente Construído, v.18, n.1, 2018. DOI: https://doi.org/10.1590/s1678$\underline{86212018000100209}$

GERALDO, B. J.; PARUSSULO, G. R.; SILVA, J. R. D.; VILATÓ, R. R.. Comparativo de custo entre alvenaria estrutural e estrutura convencional de concreto em edifícios de habitações populares. Revista Especialize, v.01, n.14, 2017.

INPE. Instituto Nacional de Pesquisas Espaciais. Monitoramento da Cobertura Florestal da Amazônia por Satélites Sistemas Prodes, Deter, Degrad e Queimadas 2007-2008. INPE, 2008.

KARPINSKI, L. A.; GUIMARÃES, J. C. B.; PANDOLFO, A.; PANDOLFO, L. M.; REINEHER, R.; KUREK, J.. Gestão Diferenciada de Resíduos da Construção Civil: Uma abordagem Ambiental. Dados eletrônicos. Porto Alegre: Edipucrs, 2009.

KATO, R. B.. Diagnosticando sistemas construtivos e empresas de construção quanto a presença de práticas 'enxutas'. Santarém, 2014.

MASCARENHAS, M. P.; ALEX, W.. Triple Bottom Line da Sustentabilidade. Revista de Administração, Contabilidade e Sustentabilidade, v.3, n.1, p.62-79, 2013.

MIRANDA, L. F. R.; ANGULO, S. C.; CARELI, É. D.. A reciclagem de resíduos de construção e demolição no Brasil: 1986 2008. Ambiente Construído, v.09, n.1, p.57-71, 2009.

MOHAMAD, G.; BAVASTRI, E. Y. N.; KIRCHHOF, L. D.; RIZZATTI, E.; JANTSCH, A. C. A.. Desenvolvimento de uma nova concepção geométrica para os blocos de concretos não modulares para alvenaria estrutural. Ambiente Construído, v.15, n.2, p.127-152, 2015. DOI: https://doi.org/10.1590/s1678-86212015000200018

KHASREEN, M. M.; BANFILL, P. F. G.; MENZIES, G. F.. Lifecycle assessment and the environmental impact of buildings: a review. Sustainability, v.1, n.3, p.674-701, 2009. DOI: 


\section{https://doi.org/10.3390/su1030674}

MURTA, A., VARUM, H., PINTO, J., BENTES, I., PAIVA, A., RAMOS, L.. Benefícios econômicos e ambientais inerentes ao uso de materiais estruturais naturais em habitações unifamiliares. Ambiente Construído, v.10, n.3, p.07-22, 2010.

PASCHOALIN FILHO, J. A.; DUARTE, E. B. L.; DIAS, A. J. G.; FARIA, A. C.. Gerenciamento dos resíduos de construção nas obras de um edifício comercial na cidade de São Paulo. Revista de Engenharia e Tecnologia, v.7, p.4, 2015.

PAIVA FILHO, J. C. P; ALMEIRA, L. A.; CASTRO, V. G.; DIODATO, M. A.. Diagnóstico do uso da madeira como material de construção no município de Mossoró-RN/Brasil. Revista Matéria, v.23, n.3, 2018. DOI: https://doi.org/10.1590/s1517-707620180003.0513

ROMAN, H.R.; ARAÚJO, H.N.; MUTTI, C.N.. Construindo em alvenaria estrutural. Florianópolis: UFSC, 1999.

SANTIAGO, A. K.. O Uso do Sistema Light Steel Framing Associado a Outros Sistemas Construtivos como Fechamento Vertical Externo Não Estrutural. Dissertação (Mestrado em Engenharia Civil) -Universidade Federal de Ouro Preto, Ouro Preto, 2008.

SILVA, D. B. M.; SALES, F. M.; SILVA, L. A.; SILVA, M. S..
Análise comparativa entre alvenaria estrutural e concreto armado. Revista Educação, Meio Ambiente e Saúde, v.7, n.4, p.103-118. 2017.

SILVA, A. T.; KERN, A. P.; PICCOLI, R.; GONZÁLEZ, M. A. S.. Novas exigências decorrentes de programas de certificação ambiental de prédios e de normas de desempenho na construção. Arquitetura Revista, v.10, n.2, p.105-114, 2014. DOI: https://doi.org/10.4013/arq.2014.102.06

SILVA, V. A.; FERNANDES, A. L.. Cenário do gerenciamento dos resíduos da construção e demolição (RCC) em UberabaMG. Revista Sociedade \& Natureza, v.24, n.2, p.333-344, 2012.

TESSARO, A. B.; SÁ, J. S.; SCREMIN, L. B.. Quantificação e classificação dos resíduos procedentes da construção civil e demolição no município de Pelotas, RS. Ambiente Construído, v.12, n.2, p.121-131, 2012. DOI: https://doi.org/10.1590/S1678-86212012000200008

ULSEN, C.; KAHN, H.; HAWLITSCHEK, G.; MASINI, E. A.; ANGULO, S. C.. Separability studies of construction and demolition waste recycled sand. Waste Management, v.33, n.3, p.656-662, 2013. DOI: https://doi.org/10.1016/j.wasman.2012.06.018

ZENID, G. J.. Madeira: uso sustentável na construção civil. 2 ed. São Paulo: Instituto de Pesquisas Tecnológicas, 2009.

A CBPC - Companhia Brasileira de Produção Científica (CNPJ: 11.221.422/0001-03) detém os direitos materiais desta publicação. Os direitos referem-se à publicação do trabalho em qualquer parte do mundo, incluindo os direitos às renovações, expansões e disseminações da contribuição, bem como outros direitos subsidiários. Todos os trabalhos publicados eletronicamente poderão posteriormente ser publicados em coletâneas impressas sob coordenação da Sustenere Publishing, da Companhia Brasileira de Produção Científica e seus parceiros autorizados. Os (as) autores (as) preservam os direitos autorais, mas não têm permissão para a publicação da contribuição em outro meio, impresso ou digital, em português ou em tradução. 